\title{
Clinical Significance of PD-L1 Expression in Brain Metastases from Non-small Cell Lung Cancer
}

\author{
SHINKICHI TAKAMORI ${ }^{1}$, GOUJI TOYOKAWA ${ }^{1}$, ISAMU OKAMOTO ${ }^{2}$, KAZUKI TAKADA $^{3}$, \\ FUMIHIKO KINOSHITA ${ }^{1}$, YUKA KOZUMA ${ }^{1}$, TAICHI MATSUBARA ${ }^{1}$, NAOKI HARATAKE ${ }^{1}$, \\ TAKAKI AKAMINE ${ }^{1}$, NOBUTAKA MUKAE ${ }^{4}$, FUMIHIKO HIRAI ${ }^{1}$, TETSUZO TAGAWA ${ }^{1}$, \\ YOSHINAO ODA ${ }^{5}$, TORU IWAKI ${ }^{6}$, KOJI IIHARA ${ }^{4}$, YOICHI NAKANISHI ${ }^{2}$ and YOSHIHIKO MAEHARA ${ }^{1}$ \\ Departments of ${ }^{1}$ Surgery and Science, ${ }^{4}$ Neurosurgery, and ${ }^{5}$ Anatomic Pathology, \\ ${ }^{2}$ Research Institute for Disease of the Chest, ${ }^{6}$ Department of Neuropathology, Neurological Institute, \\ Graduate School of Medical Sciences, Kyushu University, Higashi, Fukuoka, Japan; \\ ${ }^{3}$ Department of Thoracic Oncology, National Kyushu Cancer Center, Minami, Fukuoka, Japan
}

\begin{abstract}
Aim: To investigate the association between positivity for programmed cell death-ligand 1 (PD-L1) in brain metastases (BM) and the prognosis or clinical factors in patients with non-small cell lung cancer (NSCLC). Materials and Methods: Thirty-two patients with surgically resected brain-metastatic NSCLC were enrolled. The PD-L1 expression in $B M$ was analyzed using the antibody against human PD-L1 (clone SP142). The PD-L1 positivity was defined as PD-L1 expression on brain-metastatic tumor cells of $\geq 5 \%$. Results: Seven $(21.9 \%)$ out of 32 patients showed $P D-L 1$ positivity in BM. The PD-L1-positive BM group had a significantly shorter brain-specific disease-free survival than the PD-L1-negative BM group $(p<0.05)$. PD-LI positivity in $B M$ was significantly associated with a heavy smoking history and the administration of radiotherapy for $B M$ before surgery $(p<0.05$ and $p<0.05$, respectively). Conclusion: The PD-L1 expression in BM from NSCLC may be associated with local recurrence following surgery, and the smoking-or radiotherapy-derived effects.
\end{abstract}

Immunotherapy targeting programmed cell death-1 (PD-1) or programmed cell death-ligand 1 (PD-L1) has been widely adopted in patients with non-small cell lung cancer (NSCLC) (1). The KEYNOTE-024 study showed that select patients with NSCLC with PD-L1 positivity of $\geq 50 \%$ had a significantly

Correspondence to: Gouji Toyokawa, Department of Surgery and Science, Graduate School of Medical Sciences, Kyushu University, Fukuoka 812-8582, Japan. Tel: +81 96425466, Fax: +81 926425482, e-mail: gouji104kawa@gmail.com

Key Words: Programmed cell death-ligand 1, non-small cell lung cancer, prognosis, brain metastasis. longer median progression-free survival in the pembrolizumabtreated group than in the chemotherapy-treated group (1). Our previous study showed that PD-L1 positivity in primary lung adenocarcinoma was significantly associated with both shorter disease-free (DFS) and overall survival (OS) (2). Although assessing the expression of PD-L1 in primary NSCLC is essential for predicting the prognosis, the clinical significance of PD-L1 positivity in metastatic lesions, especially in brain metastases (BMs), is still unknown. Thus, we aimed to elucidate the relationship between PD-L1 positivity in BMs and brain-specific DFS (BS-DFS) and OS in this study.

Recently, the relationship of the PD-L1 expression in primary NSCLC and clinical factors was also investigated (2). Our previous studies showed that PD-L1 positivity in primary NSCLC was significantly associated with many clinical factors such as male sex, smoking, advanced stage, the presence of pleural and vessel invasion, and wild-type epidermal growth factor receptor $(E G F R)$ gene expression (2). However, the relationship between the PD-L1 expression in $\mathrm{BM}$ and clinical factors has not been clarified. Thus, we also investigated the association between PD-L1 positivity in BM and the clinical factors in patients with NSCLC.

\section{Materials and Methods}

Patients. From January 2005 to December 2016, 36 patients with NSCLC with BM underwent surgical resection of their brain tumors at the Department of Surgery and Science, Graduate School of Medical Sciences and Department of Neurosurgery, Kyushu University. Of these 36 patients, four who were diagnosed with small-cell lung cancer or did not have a definitive diagnosis were excluded. Of the remaining 32 patients, 15 had undergone surgical resection or a biopsy of the corresponding primary NSCLC at the Department of Surgery and Science or the Research Institute for 
Disease of the Chest, Graduate School of Medical Sciences, Kyushu University, respectively. The remaining 17 patients did not undergo surgery or biopsy for primary NSCLC. Therefore, 32 patients, including 15 cases in which both the primary and metastatic tumors were available, were enrolled in the present study.

We conducted a retrospective analysis of the age, sex, smoking status (pack-year index) and performance status (PS). Clinical or pathological $\mathrm{T}$ and $\mathrm{N}$ staging of the primary NSCLC was determined using the seventh edition of the TNM Classification of Malignant Tumors (3). The histopathological subtype, EGFR mutation status, number, size, administration of radiotherapy for BM before surgery and PD-L1 expression of the BM were also analyzed. The Institutional Review Board of our institution approved the current study (IRB No. 27-435).

Immunohistochemistry. The surgical specimens were submitted to immunohistochemical analysis in 32 cases with surgically resected BMs and in 15 cases with corresponding primary NSCLC. Formalin-fixed tissue sections were embedded in paraffin. Paraffinembedded tissue sections were dewaxed with xylene and rehydrated through a graded series of ethanol. After cutting the tissue into $4-\mu \mathrm{m}$-thick slices, endogenous peroxidase was inhibited for $30 \mathrm{~min}$ with $3 \% \mathrm{H}_{2} \mathrm{O}_{2}$ in methanol. The sections were pretreated in a decloaking chamber with Target Retrieval Solution (Dako, Glostrup, Denmark) for $15 \mathrm{~min}$ at $110^{\circ} \mathrm{C}$, and incubated with a monoclonal antibody at $4^{\circ} \mathrm{C}$ overnight. The immune complex was detected by a DAKO EnVision Detection System (Dako). The sections were reacted in 3,3'-diaminobenzidine, and counterstained with hematoxylin. All of the slides were dehydrated in a graded series of alcohol and xylene. They were then mounted and cover slips were applied.

A rabbit monoclonal antibody to human PD-L1 (clone SP142, dilution 1:100; Spring Bioscience, Ventana, Tucson, AZ, USA) was used as the primary antibody (2). The evaluation of PD-L1 expression was independently performed by a surgeon (S.T.) and a pathologist (K.T.). The percentage of PD-L1-positive tumor cells was determined as four categories: $<1 \% ; 1 \%-4 \% ; 5 \%-49 \%$; and $50 \%-100 \%$. Lesions with PD-L1 expression in $\geq 5 \%$ of total tumor cells were considered positive (4). Human placental sections were used as negative and positive controls.

Statistical analyses. The association between PD-L1 expression and clinical factors was analyzed using two-sided Fisher's exact test, Student's $t$-test and Mann-Whitney $U$-test where appropriate. The BS-DFS and OS were defined as the time (months) from the day of surgery until brain recurrence and death, respectively. The survival probabilities were estimated using the Kaplan-Meier method and log-rank test. The differences were considered to be statically significant when the $p$-value was less than 0.05 . All of the statistical analyses were performed using the $\mathrm{JMP}^{\circledR} 13$ software program (SAS Institute Inc., Cary, NC, USA).

\section{Results}

Characteristics of the patients enrolled in the current study. Table I shows the characteristics of the patients included in this study. The majority of patients $(71.9 \%)$ were current or former smokers, and many $(62.5 \%)$ had a smoking history of $\geq 40$ pack-years. Multiple BMs were observed in 14
Table I. The characteristics of 32 patients with non-small cell lung cancer (NSCLC) with brain metastasis (BM).

\begin{tabular}{|c|c|}
\hline Factor & Value/no. of patients \\
\hline \multicolumn{2}{|l|}{ Age (years) } \\
\hline Median (range) & $64(29-76)$ \\
\hline \multicolumn{2}{|l|}{ Gender } \\
\hline Male & 21 \\
\hline Female & 11 \\
\hline \multicolumn{2}{|l|}{ Smoking status } \\
\hline Never-smoker & 9 \\
\hline Smoker & 23 \\
\hline \multicolumn{2}{|l|}{ Pack-years } \\
\hline$<40$ & 12 \\
\hline$\geq 40$ & 20 \\
\hline \multicolumn{2}{|l|}{ Performance status } \\
\hline 0 & 7 \\
\hline 1 & 11 \\
\hline 2 & 8 \\
\hline 3 & 6 \\
\hline \multicolumn{2}{|l|}{ T-Stage } \\
\hline 1 & 11 \\
\hline 2 & 8 \\
\hline 3 & 8 \\
\hline 4 & 5 \\
\hline \multicolumn{2}{|l|}{$\mathrm{N}$-Stage } \\
\hline 0 & 11 \\
\hline 1 & 5 \\
\hline 2 & 12 \\
\hline 3 & 4 \\
\hline \multicolumn{2}{|l|}{ Number of BMs } \\
\hline Single & 18 \\
\hline Multiple & 14 \\
\hline \multicolumn{2}{|l|}{ Size of BMs $(\mathrm{cm})$} \\
\hline Mean (SD) & $3.5(1.3)$ \\
\hline \multicolumn{2}{|l|}{ Pathological subtype } \\
\hline Adenocarcinoma & 30 \\
\hline Squamous cell carcinoma & 2 \\
\hline \multicolumn{2}{|l|}{ EGFR mutation } \\
\hline None & 24 \\
\hline L858R & 3 \\
\hline 19 deletion & 5 \\
\hline \multicolumn{2}{|l|}{$\begin{array}{l}\text { Systemic chemotherapy for } \\
\text { primary NSCLC before surgery }\end{array}$} \\
\hline None & 26 \\
\hline Platinum-based regimen & 4 \\
\hline Others & 2 \\
\hline \multicolumn{2}{|c|}{ Radiotherapy for BM before surgery } \\
\hline None & 28 \\
\hline Stereotactic radiosurgery & 4 \\
\hline \multicolumn{2}{|c|}{ PD-L1 expression in primary NSCLC } \\
\hline$<1 \%$ & 10 \\
\hline $1-4 \%$ & 1 \\
\hline $5-49 \%$ & 2 \\
\hline$>50 \%$ & 2 \\
\hline \multicolumn{2}{|l|}{ PD-L1 expression in BM } \\
\hline$<1 \%$ & 24 \\
\hline $1-4 \%$ & 1 \\
\hline $5-49 \%$ & 2 \\
\hline$>50 \%$ & 5 \\
\hline
\end{tabular}

SD, Standard deviation; EGFR, epidermal growth factor receptor; PD-L1, programmed cell death-ligand 1. 
A

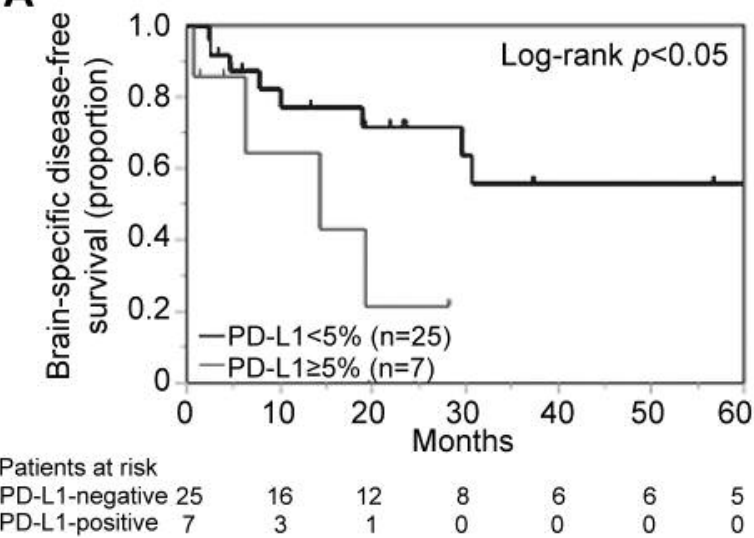

B

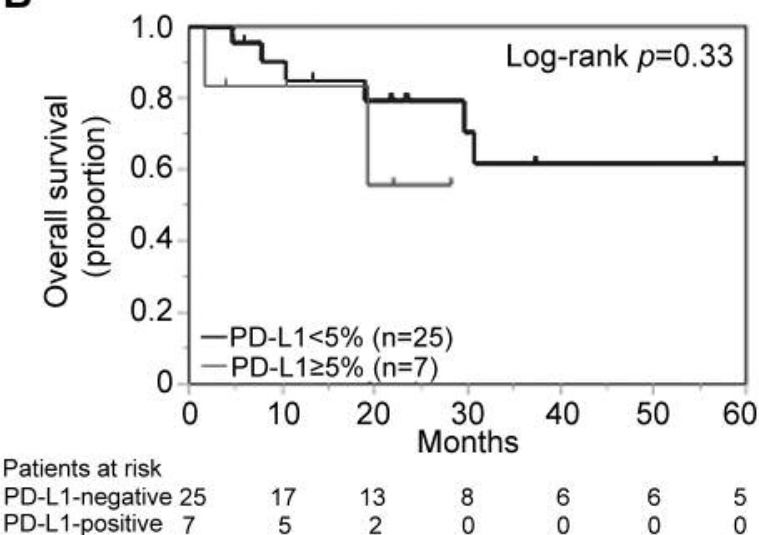

Figure 1. Kaplan-Meier curves showing brain-specific disease-free survival (A) and the overall survival (B) of patients with non-small cell lung cancer according to the expression of programmed cell death-ligand 1 in brain metastases (cut-off: $5 \%$ ).

Table II. The characteristics of patients with non-small cell lung cancer (NSCLC) with programmed cell death-ligand 1 (PD-L1) positivity in brain metastases (BM) (cut-off: 5\%).

PD-L1 in BM

\begin{tabular}{|c|c|c|c|c|}
\hline & & & & \\
\hline Factor & $\mathrm{n}=32$ & Negative $(n=25)$ & Positive $(n=7)$ & $p$-Value \\
\hline Age (years) & Mean (SD) & $61(2.4)$ & $60(4.6)$ & $0.749^{\mathrm{a}}$ \\
\hline Gender, $\mathrm{n}$ & Male & 15 & 6 & $0.374^{\mathrm{b}}$ \\
\hline & Female & 10 & 1 & \\
\hline Smoking history (pack-years) & Median (range) & $19(0-63)$ & $66(5-84)$ & $0.014^{\mathrm{c}}$ \\
\hline Performance status, $\mathrm{n}$ & $\leq 1$ & 14 & 4 & $1.000^{\mathrm{b}}$ \\
\hline & $\geq 2$ & 11 & 3 & \\
\hline EGFR mutation, $\mathrm{n}$ & Wild-type & 19 & 5 & $1.000^{\mathrm{b}}$ \\
\hline & Sensitive & 6 & 2 & \\
\hline Systemic chemotherapy for primary NSCLC before surgery, $\mathrm{n}$ & None & 22 & 4 & $0.101^{\mathrm{b}}$ \\
\hline & Chemotherapy & 3 & 3 & \\
\hline Radiotherapy for BM before surgery, $\mathrm{n}$ & None & 24 & 4 & $0.025^{\mathrm{b}}$ \\
\hline & Stereotactic radiosurgery & 1 & 3 & \\
\hline PD-L1 positivity in primary NSCLC, $\mathrm{n}^{*}$ & Positive & 2 & 2 & $0.517^{\mathrm{b}}$ \\
\hline & Negative & 9 & 2 & \\
\hline
\end{tabular}

SD, Standard deviation; EGFR, epidermal growth factor receptor. *Cases in which specimens of both the primary and metastatic tumors were available $(\mathrm{n}=15)$. ${ }^{\text {aS }}$ Student's $t$-test; b bo-sided Fisher's exact test; 'Mann-Whitney $U$-test.

$(43.8 \%)$ patients. The majority of patients $(93.7 \%)$ were pathologically diagnosed with adenocarcinoma and squamous cell carcinoma, respectively. A sensitive EGFR mutation was detected in eight $(25.0 \%)$ patients. No patients received molecular-targeted agents before surgical resection of BM, while six $(18.8 \%)$ underwent chemotherapy prior to surgery. Four (12.5\%) underwent radiotherapy for their BM followed by surgical resection. Four $(26.7 \%)$ and seven (21.9\%) patients presented PD-L1-positive primary and metastatic sites, respectively (4).
Impact of PD-L1 positivity in BM on the BS-DFS and OS. The PD-L1-positive BM group had a significantly shorter BSDFS than the PD-L1-negative BM group (64\% vs. $77 \%$ at 1 year, and $21 \% v s .71 \%$ at 2 years; $p<0.05)$. No significant difference in the OS was observed between the PD-L1positive and -negative BM groups $(p=0.33)$. The KaplanMeier curves are shown in Figure 1. PD-L1 positivity in primary NSCLC was not significantly associated with BSDFS $(p=0.100)$, although the sample size was small $(n=15$, data not shown). 
Relationship between PD-L1 positivity in BM and clinical factors. Table II shows the relationship between PD-L1 positivity in $\mathrm{BM}$ and clinical factors. A heavy smoking history and the administration of radiotherapy for BM before surgery showed a significant trend toward association with PD-L1 positivity in BM $(p<0.05$ and $p<0.05$, respectively). In contrast, age, sex, PS, EGFR mutation status, systemic chemotherapy before surgery and PD-L1 positivity in primary NSCLC was not significantly associated with PDL1 expression in BMs $(p=0.749, p=0.374, p=1.000$, $p=1.000, p=0.101$, and $p=0.517$, respectively).

\section{Discussion}

To the best of our knowledge, this is the first report to show the clinical significance of the PD-L1 positivity in metastatic lesions. In the current study, we found PD-L1-positive BM in $21.9 \%$ of the 32 cases using the clone SP142 antibody. Mansfield et al. reported PD-L1-positive BM in approximately $32.9 \%$ of 73 cases using clone E1L3N antibody with the cutoff value at $5 \%$ (4). Our results were almost consistent with their report, taking into account the lower sensitivity of the SP142 clone reported by the Blueprint study (5). As shown in Figure 1, the PD-L1-positive BM group had a significantly shorter BS-DFS than the PD-L1-negative BM group. Given that the KEYNOTE-024 study revealed that select patients with NSCLC with at least 50\% PD-L1 positivity in their primary lung cancer had a significantly longer progressionfree survival in the pembrolizumab-treated group than in the chemotherapy-treated group (1), the analysis of PD-L1 positivity in BM may be useful for deciding postoperative therapeutic strategies or the follow-up interval period.

Because of the anatomical location of the samples, it is often difficult to investigate the PD-L1 expression in BM before treatment. With regard to the concordance rates of PD-L1 positivity between primary NSCLC and the corresponding BM, the current study and that of Mansfield et al. (4) showed that PD-L1 positivity in primary lesions was consistent with that of metastatic tumors in $75 \%$ and $86.2 \%$ of the total cases, respectively. Thus, the PD-L1 positivity in primary NSCLC may be sometimes be misleading for predicting the PD-L1 expression in BM (6). Furthermore, a recent report by Suda et al. using autopsy specimens showed that the heterogeneity of the expression of PD-L1 was dependent on the metastatic site (7). In the present study, a heavy smoking status and radiotherapy for the BM, but not the PD-L1 expression in primary NSCLC, were significantly associated with the PD-L1 positivity in BM (Table I). Although the sample size analyzed in this study was very small, substantial evidence exists to support the association between PD-L1 positivity and smoking status or radiotherapy. With regard to cigarette smoking, lung cancer in smokers was reported to be associated with a high burden of neoantigens, increased immunogenicity and upregulation of PD-L1 (8-11). Regarding the relationship between PD-L1 expression and radiotherapy, pre-clinical studies have shown that PD-L1 expression was up-regulated both in vivo and in vitro after radiotherapy (12-14). In addition, some experimental studies have suggested that radiotherapy mediated the recruitment of $\mathrm{CD}^{+}$T-cells, which was reported to be associated with the intra-tumoral PD-L1 expression $(15,16)$. The results of those reports partly support our present findings.

The present study is associated with some limitations. One limitation is that this study investigated a small cohort of patients. Although the samples of BMs from NSCLC are rarely available, the small number of the cases makes it difficult to draw definite conclusions. Another limitation is that the PD-L1 analysis was performed using only one antibody (SP142). According to a previous report, the positivity rate using SP142 was found to be lower than that of other antibodies such as 28-8, 22C3 and SP263 (5). However, the rate of SP142-positivity was reported to be higher than that of other antibodies such as 28-8 and E1L3N in patients with small-cell lung cancer (17). Therefore, the PD-L1-positivity should be evaluated using other antibodies or cut-off values in future studies.

In conclusion, the expression of PD-L1 in BM was significantly associated with a shorter BS-DFS. A heavy smoking history and radiotherapy for BM before surgery were significantly associated with PD-L1 positivity in BM. These findings should be validated in future prospective studies with a larger patient population.

\section{Acknowledgements}

None.

\section{Conflicts of Interest}

Yoichi Nakanishi has received honoraria and research funding from Chugai Pharmaceutical Co. Ltd. Isamu Okamoto has received honoraria from Chugai Pharmaceutical Co. Ltd. All remaining Authors declare no conflicts of interest in regard to this study.

\section{References}

1 Reck M, Rodriguez-Abreu D, Robinson AG, Hui R, Csoszi T, Fulop A, Gottfried M, Peled N, Tafreshi A, Cuffe S, O'Brien M, Rao S, Hotta K, Leiby MA, Lubiniecki GM, Shentu Y, Rangwala $\mathrm{R}$ and Brahmer JR: Pembrolizumab versus chemotherapy for pd-11-positive non-small-cell lung cancer. $\mathrm{N}$ Engl J Med 375: 1823-1833, 2016.

2 Takada K, Okamoto T, Shoji F, Shimokawa M, Akamine T, Takamori S, Katsura M, Suzuki Y, Fujishita T, Toyokawa G, Morodomi Y, Okano S, Oda Y and Maehara Y: Clinical significance of PD-L1 protein expression in surgically resected primary lung adenocarcinoma. J Thorac Oncol 11: 1879-1890, 2016. 
3 Boffa DJ and Greene FL: Reacting to changes in staging designations in the 7 th edition of the AJCC staging manual. Annals of surgical oncology 18: 1-3, 2011.

4 Mansfield AS, Aubry MC, Moser JC, Harrington SM, Dronca RS, Park SS and Dong H: Temporal and spatial discordance of programmed cell death-ligand 1 expression and lymphocyte tumor infiltration between paired primary lesions and brain metastases in lung cancer. Ann Oncol 27: 1953-1958, 2016.

5 Hirsch FR, McElhinny A, Stanforth D, Ranger-Moore J, Jansson M, Kulangara K, Richardson W, Towne P, Hanks D, Vennapusa B, Mistry A, Kalamegham R, Averbuch S, Novotny J, Rubin E, Emancipator K, McCaffery I, Williams JA, Walker J, Longshore J, Tsao MS and Kerr KM: PD-L1 immunohistochemistry assays for lung cancer: results from phase 1 of the Blueprint PD-L1 IHC Assay Comparison Project. J Thorac Oncol 12: 208-222, 2017.

6 Takamori S, Toyokawa G, Okamoto I, Takada K, Kozuma Y, Matsubara T, Haratake N, Akamine T, Katsura M, Mukae N, Shoji F, Okamoto T, Oda Y, Iwaki T, Iihara K, Nakanishi Y and Maehara Y: Discrepancy in programmed cell death-ligand 1 between primary and metastatic non-small cell lung cancer. Anticancer Res 37: 4223-4228, 2017.

7 Suda K, Murakami I, Yu H, Kim J, Ellison K, Rivard CJ, Mitsudomi $\mathrm{T}$ and Hirsch FR: Heterogeneity in immune marker expression after acquisition of resistance to EGFR kinase inhibitors: analysis of a case with small cell lung cancer transformation. J Thorac Oncol 12: 1015-1020, 2017.

8 Inamura K, Yokouchi Y, Kobayashi M, Sakakibara R, Ninomiya $\mathrm{H}$, Subat S, Nagano H, Nomura K, Okumura S, Shibutani T and Ishikawa Y: Tumor B7-H3 (CD276) expression and smoking history in relation to lung adenocarcinoma prognosis. Lung Cancer 103: 44-51, 2017.

9 Sacher AG and Gandhi L: Biomarkers for the clinical use of PD1/PD-L1 inhibitors in non-small-cell lung cancer: a review. JAMA Oncol 2: 1217-1222, 2016.

10 Strickland KC, Howitt BE, Shukla SA, Rodig S, Ritterhouse LL, Liu JF, Garber JE, Chowdhury D, Wu CJ, D'Andrea AD, Matulonis UA and Konstantinopoulos PA: Association and prognostic significance of BRCA1/2-mutation status with neoantigen load, number of tumor-infiltrating lymphocytes and expression of PD-1/PD-L1 in high grade serous ovarian cancer. Oncotarget 7: 13587-13598, 2016.
11 Takada K, Toyokawa G, Okamoto T, Shimokawa M, Kozuma Y, Matsubara T, Haratake N, Akamine T, Takamori S, Katsura M, Shoji F, Oda Y, and Maehara Y: A Comprehensive analysis of programmed cell death ligand-1 expression with the clone SP142 antibody in non-small-cell lung cancer patients. Clinical lung cancer 18: 572-582, 2017.

12 Gong X, Li X, Jiang T, Xie H, Zhu Z, Zhou F, and Zhou C: Combined radiotherapy and anti-PD-L1 antibody synergistically enhances antitumor effect in non-small cell lung cancer. J Thorac Oncol 12: 1085-1097, 2017.

13 Herter-Sprie GS, Koyama S, Korideck H, Hai J, Deng J, Li YY, Buczkowski KA, Grant AK, Ullas S, Rhee K, Cavanaugh JD, Neupane NP, Christensen CL, Herter JM, Makrigiorgos GM, Hodi FS, Freeman GJ, Dranoff G, Hammerman PS, Kimmelman AC and Wong KK: Synergy of radiotherapy and PD-1 blockade in KRAS-mutant lung cancer. JCI insight 1: e87415, 2016.

14 Takamori S, Toyokawa G, Takada K, Shoji F, Okamoto T and Maehara Y: Combination therapy of radiotherapy and anti-PD1/PD-L1 treatment in non-small-cell lung cancer: a mini-review. Clin Lung Cancer 2017. doi: 10.1016/j.cllc.2017.06.015. [Epub ahead of print]

15 Lee Y, Auh SL, Wang Y, Burnette B, Wang Y, Meng Y, Beckett M, Sharma R, Chin R, Tu T, Weichselbaum RR and Fu YX: Therapeutic effects of ablative radiation on local tumor require CD8+ T-cells: changing strategies for cancer treatment. Blood 114: 589-595, 2009.

16 Lim JY, Gerber SA, Murphy SP and Lord EM: Type I interferons induced by radiation therapy mediate recruitment and effector function of CD8(+) T-cells. Cancer Immunol Immunother 63: 259-271, 2014.

17 Takada K, Toyokawa G, Okamoto T, Akamine T, Takamori S, Katsura M, Fujishita T, Shoji F, Oda Y and Maehara Y: An immunohistochemical analysis of PD-L1 protein expression in surgically resected small cell lung cancer using different antibodies and criteria. Anticancer Res 36: 3409-3412, 2016.

Received October 24, 2017

Revised November 9, 2017

Accepted November 13, 2017 